\title{
Numerical Study on Spoiler Torque Effect to a Half Buried Pipeline
}

\author{
H.C. Zheng, B. Shi, Q. Yu \\ College of Engineering \\ Ocean University of China \\ Qingdao
}

\begin{abstract}
The kinetics of flow around submarine pipeline with spoiler is studied by lattice Boltzmann method. The pipeline is half buried in the seabed. The existence of spoiler not only increases the contact pressure difference along seabed surface, but also leads to additional torque acting on the pipeline center. The latter has certain potential safety hazard to pipeline. Present study gives torque level under different spoiler height and the corresponding mechanism is illustrated.
\end{abstract}

Key words-LBM; submarine pipeline; spoiler; torque; pressure difference

\section{INTRODUCTION}

The submarine pipeline scour is a common phenomenon in ocean engineering and corresponding protection is necessary. The traditional protection includes two basic ideas. One idea is to avoid scour starting [1,2], and the other one is to accelerate scour in order to reach self-buried happening [3]. Hulsbergen $[4,5]$ found that a rigid spoiler on the top of the buried pipeline can accelerate the buried process speed up to 10 times. Han [6] and Zhang [7] made a further experimental study and gave appropriate spoiler height based on scour starting. But no one studied the spoiler torque effect of a submarine pipeline which is the focus of this paper.

\section{LATTICE BOLTZMANN METHOD}

Lattice Boltzmann method (LBM) is a mesoscopic numerical method which has developed more than twenty years and achieved great success in simulating a wide variety of physical systems. LBM has advantages of easy programming, parallel computing, and easy handling of complex boundary conditions, etc. [8].

\section{A. Lattice Boltzmann Equation}

Two-dimensional nine-velocity model (D2Q9) is adopted in our simulation. The standard lattice Boltzmann Bhatnagar-Gross-Krook (LBGK) equation is [8]

$$
f_{\alpha}\left(\mathbf{x}+\mathbf{e}_{\alpha} \delta_{t}, t+\delta_{t}\right)-f_{\alpha}(\mathbf{x}, t)=-\frac{1}{\tau}\left[f_{\alpha}(\mathbf{x}, t)-f_{\alpha}^{e q}(\mathbf{x}, t)\right]
$$

Where $f_{\alpha}(\mathbf{x}, t)$ and $f_{\alpha}^{e q}(\mathbf{x}, t)$ are the particle distribution function and equilibrium distribution function respectively at $\alpha t h$ direction, $\delta_{t}$ denotes lattice time step and $\tau$ represents the dimensionless relaxation time. The equilibrium distribution function $f_{\alpha}^{e q}(\mathbf{x}, t)$ is

$$
\left.f_{\alpha}^{e q}(\mathbf{x}, t)=\omega \rho 1+\frac{3}{c^{2}}\left(\mathbf{e}_{\alpha} \cdot \mathbf{u}\right)+\frac{9}{c^{4}}\left(\mathbf{e}_{\alpha} \cdot \mathbf{u}\right)^{2}-\frac{3}{2 c^{2}} \mathbf{u}^{2}\right]
$$

The macroscopic variables $\rho, \mathbf{u}$ can be linearly described by distribution function:

$$
\rho=\sum_{\alpha} f_{\alpha}, \quad \rho \mathbf{u}=\sum_{\alpha} \mathbf{e}_{\alpha} f_{\alpha}
$$

\section{B. Spoiler Torque Calculation}

The torque on a point of the spoiler can be calculated by the force that fluid acting on the spoiler multiplying the arm. Then total torque can be obtained by a sum calculation.

$$
\mathbf{T}=\sum_{\text {all } \mathrm{x}_{w}} \mathbf{r}\left(x_{w}\right) \times \mathbf{F}\left(x_{w}\right)
$$

Here, $x_{w}$ is a point of the spoiler. The arm $\mathbf{r}\left(x_{w}\right)$ is the distance of boundary point to the pipeline center. The force $\mathrm{F}\left(x_{w}\right)$ is calculated by bounce-back method [8].

\section{INTRODUCTION OF THE MODEL}

In present work, the pipeline is half buried in seabed. A spoiler is installed on the top of the pipeline. The spoiler height is $h$ and pipeline radius is $r(r=20)$. Then pipeline diameter $D=40$. The inlet velocity is $U_{\infty}\left(U_{\infty}=0.5\right)$. Point A and $\mathrm{B}$ are two contact points of pipeline and seabed. Point $\mathrm{A}$ is upstream contact point and point B is downstream contact point.

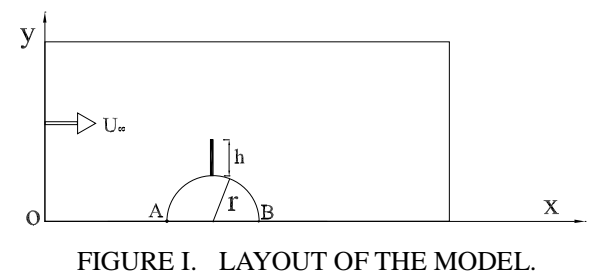

\section{NUMERICAL RESULTS AND ANALYSIS}

The ratio of spoiler height relative to pipeline radius is from 0.1 to 2.0. The pressure difference $p_{A B}\left(p_{A B}=p_{A}-p_{B}\right)$ and torque under different spoiler height is listed in tab. 1. Pressure difference and torque increase while spoiler height 
increasing. Pressure difference increases slowly. Yet torque increases relatively quickly. It should be pointed out that present results are of per unit pipeline length. For a long enough pipeline, torque can't be ignored when spoiler height reach to a certain value, e.g. $h / r \geq 1.5$.

TABLE I .PRESSURE DIFFERENCE AND TORQUE UNDER DIFFERENT SPOILER HEIGHT.

\begin{tabular}{|c|c|c|c|c|}
\hline $\mathbf{h} / \mathbf{r}$ & 0.1 & $\begin{array}{c}0.2 \\
5\end{array}$ & 0.5 & $\begin{array}{c}0.7 \\
5\end{array}$ \\
\hline $\begin{array}{c}\mathbf{p}_{\mathrm{AB}} \\
\left(\times \mathbf{1 0}^{-2}\right. \\
)\end{array}$ & $\begin{array}{l}9 . \\
72\end{array}$ & $\begin{array}{c}10 . \\
0 .\end{array}$ & $\begin{array}{l}10 . \\
02\end{array}$ & \\
\hline $\begin{array}{c}\text { Torqu } \\
\text { e } \\
\left(\times 10^{-4}\right. \\
)\end{array}$ & 0 & $\begin{array}{c}0 . \\
028\end{array}$ & $\begin{array}{c}0 . \\
081\end{array}$ & \\
\hline & & & & \\
\hline
\end{tabular}

(a)

\begin{tabular}{|c|c|c|c|c|}
\hline 1.0 & $\begin{array}{c}1.2 \\
5\end{array}$ & 1.5 & $\begin{array}{c}1.7 \\
5\end{array}$ & 2.0 \\
\hline 10. & 10. & 10. & 10. & 10. \\
\hline 34 & 51 & 59 & 62 & 67 \\
\hline 0. & 0. & 3. & 28. & 131 \\
\hline 246 & 820 & 719 & 0 & .0 \\
\hline \multirow{2}{*}{\multicolumn{5}{|c|}{0}} \\
\hline & & & & \\
\hline & & & & \\
\hline & & & & \\
\hline & & & & \\
\hline
\end{tabular}

(b)
FIGURE II. THE INCREASING TREND OF (A): PRESSURE DIFFERENCE AND (B): TORQUE.

We can further find the increasing trend of pressure difference and torque with spoiler height increasing from Figure. 2.. When $h / r \geq 1.5$, pressure difference increases more slowly and torque increases faster.

Figure. 3. shows the streamlines under different spoiler height. When $h / r \leq 0.25$, there is only one eddy at downstream. When $h / r=0.5$, there is one new eddy began to appear near the seabed. When spoiler height continues increasing, the new generated eddy begins to grow. Two eddies reach to similar size when $h / r$ is more than 1.0.

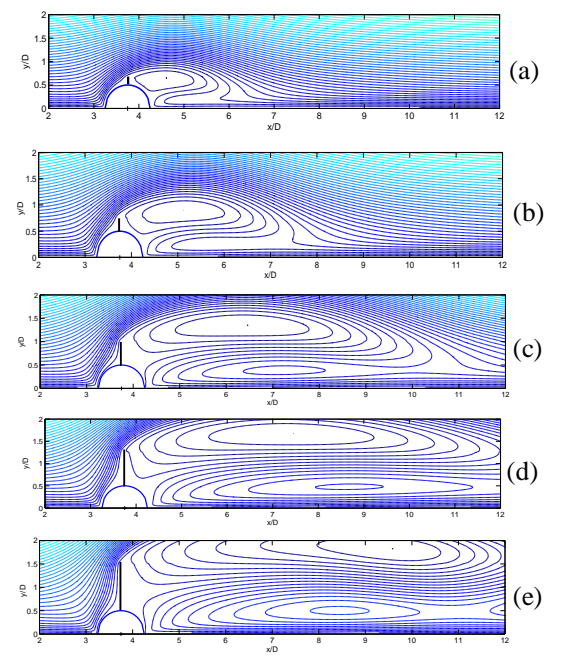

FIGURE III. STREAMLINES UNDER $h / r=$ (A) 0.25 ; (B) 0.5 ; (C) 1.0 ; (D) 1.5 ; (E) 2.0 .

The spoiler is collided by fluids on both sides of upstream and downstream. The downstream is a low pressure space and the force of fluid acted on the spoiler is smaller than that of upstream. The higher the spoiler is, the bigger pressure difference is. So torque increases rapidly.

The contact point B is far from the spoiler top. So the increase of spoiler height has little influence on the pressure of point $B$. In addition, the pressure of point $A$ almost remains the same. Then the pressure difference of point A and B increases slowly.

\section{V.CONCLUSION}

The torque effect of the spoiler on a half buried pipeline is numerical studied. The influence of spoiler height increasing on pressure difference and torque is obtained. When spoiler height increases, pressure difference increases slowly, but torque increases rapidly when $h / r \geq 1.5$. For a long enough pipeline, the torque effect can't be ignored when spoiler height is more than a certain value.

\section{ACKNOWLEDGEMENT}

This study was financially supported by National Nature Science Fund of China(Grant No. 51279189; 50879084).

B. Shi, corresponding author, sediment@ouc.edu.cn

H.C. Zheng,

zhenghaicheng@ouc.edu.cn)

6220941@qq.com (or

\section{REFERENCES}

[1] D.Y. Zhao, J.X. Yu, G.X. Li, et. al.. An experimental scour prevention technique for subsea pipelines. Journal of Harbin Engineering University, 30(6), pp. 597-601, 2009.

[2] G.L. Yu, Q.Q. Chen, Y.H. Li. Status and tendency of development of scour prevention technique for submarine pipeline. Water Resources and Hydropower Engineering, 38(11), pp. 30-33, 2007.

[3] Y.M. Chiew . Effect of spoilers on scour at submarine pipelines. Journal of Hydraulic Engineering, 118( 9), pp. 1311-1317, 1992.

[4] C.H. Hulsbergen. Spoilers for stimulated burial of submarine pipelines. Proc. Of the 18th Conf. on Offshore Technol, 5339, pp. 441-444, 1986.

[5] C.H. Hulsbergen, R. Bijker. Effect of spoilers on submarine pipeline stability. Conf. of 21st Annual Offshore Technology, 6154, pp. 337-350, 19889.

[6] Y. Han, B. Shi, X.Y. Ren, et al.. Experimental study on the function of spoilers in protecting submarine pipeline. Journal of Sichuan University (Engineering Science Edition), 42(2), pp. 100-106, 2010.

[7] Z.Y. Zhang, B. Shi, F.F. Fan, et. al.. Numerical simulation and experimental study on flow around submarine pipeline with spoiler. Chinese Journal of Hydrodynamics, 26(5), pp. 597-605, 2011.

[8] H.C. Zheng, F.F. Wu. Lattice Boltzmann simulation of flow past a circular cylinder near a moving wall. International Journal for Numerical Methods in Fluids, 69(11), pp. 1753-1761, 2012. 